\title{
The Association between Myopia and Primary Open-Angle Glaucoma: A Systematic Review and Meta-Analysis
}

\author{
Jian Wu ${ }^{a}$ Jie Hao ${ }^{a, b}$ Yifan Du ${ }^{a}$ Kai Cao ${ }^{a} b$ Caixia Lin ${ }^{a}$ Runzhou Sun ${ }^{a}$ \\ Yuan Xie $^{a}$ Ningli Wang ${ }^{a, b}$ \\ aBeijing Tongren Eye Center, Beijing Tongren Hospital, Beijing Key Laboratory of Ophthalmology and \\ Visual Sciences, Capital Medical University, Beijing, China; ${ }^{b}$ Beijing Institute of Ophthalmology, Beijing, China
}

\section{Keywords}

Myopia $\cdot$ Primary open-angle glaucoma $\cdot$ Systematic

review $\cdot$ Meta-analysis

\begin{abstract}
Background: Previous studies identified myopia as a risk factor for primary open-angle glaucoma (POAG). However, recent studies have shown different results, and the definitive relationship between myopia and POAG remains controversial. Objectives: The aim of this study was to investigate the relationship between myopia and POAG. Methods: Published articles were searched from PubMed, Embase, and Scopus databases between 1970 and 2020. A pooled analysis of the odds ratios (ORs) was performed using a randomeffects model. Results: Data on the association between myopia and POAG were obtained from 16 cross-sectional studies, and the pooled OR was 2.26 (95\% confidence interval $[C l], 1.77-2.89, p<0.001)$ in random-effects model $\left(I^{2}=86 \%\right.$; $p<0.01)$. For the relationship of myopia and POAG progression, data from 7 longitudinal cohort studies were included, and the pooled OR was $0.85(95 \% \mathrm{Cl}, 0.73-0.99, p=0.042)$ in
\end{abstract}

the random-effects model $\left(I^{2}=88 \% ; p<0.01\right)$. Conclusion: Our findings demonstrated that myopia may be a risk factor associated with POAG and a possible protective factor for POAG progression. It may be due to myopia with the presence of a lamina cribrosa defect, slowing down the visual field loss and also POAG progression. Further research for underlying mechanisms is still needed.

(C) 2021 The Author(s) Published by S. Karger AG, Basel

\section{Introduction}

Myopia is one of the most prevalent eye disorders that have become the major leading cause of blindness worldwide [1]. The direct cost of vision loss was estimated to be more than 2 trillion US dollars globally in 2012 [2]. Myopia is always associated with comorbidities that increase the risks of severe and irreversible loss of vision, such as retinal detachment, subretinal neovascularization, dense

Jian Wu and Jie Hao contributed equally to the work and should be regarded as co-first authors.
C 2021 The Author(s).

Published by S. Karger AG, Basel

This is an Open Access article licensed under the Creative Common Attribution-NonCommercial-4.0 International License (CC BY-NC) (http://www.karger.com/Services/OpenAccessLicense), applicable to the online version of the article only. Usage and distribution for commercial purposes requires written permission.
Correspondence to:

Ningli Wang,wningli@vip.163.com 
cataract, and glaucoma [3-5]. Of these, glaucoma is a major comorbidity. Primary open-angle glaucoma (POAG) refers to slowly progressive atrophy of the optic nerve, characterized by a loss of peripheral visual function and an excavated appearance of the optic disc [6]. There is currently no curative therapy available for POAG [7]. Based on findings from previous cross-sectional studies and a meta-analysis, myopia is considered to be a risk factor for POAG [8].

In 1925, Knapp et al. [9] reported that POAG might be associated with myopia. Thereafter, numerous demographic studies investigated the relationship between myopia and POAG, with contradicting results [10-25]. Some researchers proposed that myopia was an independent risk factor for POAG $[15,16,19,25]$; nevertheless, the findings of another study [13] suggested that only high myopia was a risk factor for POAG, and other authors even reported no association between the degree of myopia and the prevalence of POAG [26]. Moreover, the etiology of myopia [27], age, intraocular pressure (IOP), and axial length (AL) may be confounders, affecting the correlation analysis between myopia and POAG [28-31].

Recently, a large number of longitudinal cohort studies have shown that myopia is not a risk factor for the progression of POAG [26, 32-34], and the relevant results even indicated that myopia may be a protective factor for POAG progression [33]. The lamina cribrosa defect may play a role in myopia-related POAG $[35,36]$. These previous researchers pointed out that the lamina cribrosa was significantly thinner in myopic (and especially in high myopic) eyes, which could not tolerate the translaminar pressure gradient. These findings provide a plausible explanation for the increased susceptibility to glaucoma in patients with myopia. However, the definitive relationship between myopia and POAG remains controversial.

The purpose of this study was to investigate the relationship between myopia and POAG. We reviewed articles from 2 perspectives: (1) myopia and POAG in crosssectional studies and (2) myopia and the progression of POAG in cohort studies.

\section{Methods}

\section{Search Strategy}

Three ophthalmologists (J.W., J.H., and R.Z.S.) independently performed the literature retrieval. A systematic review of the literature from PubMed, Embase, and Scopus databases from January 1, 1970, to October 31, 2020, was conducted according to the MOOSE guidelines [37]. The retrieval process was de- signed to include "myopia and POAG" from cross-sectional studies and "myopia and the progression of POAG" from cohort studies.

The search terms used in PubMed included were shown in online supplementary Material 1 (for all online suppl. material, see www.karger.com/doi/10.1159/000520468). Articles retrieved from the PubMed and Embase databases were imported into EndNote software (version 8.1), where duplicate articles were deleted manually. Titles and abstracts of the remaining studies were scanned by 3 ophthalmologists (J.W., J.H., and R.Z.S.) independently. The extracted studies were compared, and inconsistencies were resolved by consensus. Full texts were reviewed, and the reference lists extracted from the articles were checked.

\section{Inclusion and Exclusion Criteria}

Studies were included if they (1) had a clear classification standard for myopia, (2) had POAG or progression of POAG as the outcome event, and (3) evaluated the association using either odds ratio (OR) or with $95 \%$ confidence interval (CI) or allowed for the calculation of the OR from the raw data presented in the article or correlation coefficient value, a regression coefficient value. The exclusion criteria included ( 1$) \leq 18$ years old, (2) an unmatched study type, such as case report and review, (3) studies involving secondary glaucoma or angle-closure glaucoma, (4) studies published in a language other than English, (5) studies without a clear-cut definition of myopia, (6) studies without a detailed description of POAG or progression of POAG, or (7) patient who converted from ocular hypertension to OAG in the course of progress (follow-up). When multiple publications from the same study population were available, we checked for duplicate analysis and included only the most recent publication.

\section{Data Extraction and Quality Assessment}

The following characteristics were extracted: (1) last name of the first author, (2) year of publication, (3) study design, (4) ethnicity of the study population, (5) number of subjects in the analysis, (6) age range of subjects, (7) definition of POAG or progression of POAG, (8) definition of myopia or mean spherical equivalent, (9) effect estimate, (10) adjusted confounding factors, and (11) mean follow-up period.

\section{Statistical Analysis}

We combined the study-specific ORs from the cross-sectional studies and longitudinal cohort studies to estimate the pooled OR with 95\% CI using the random-effects model separately. The ORs were compared between myopia $(\geq-0.5 \mathrm{D})$ and the nonmyopia group $(<-0.5 \mathrm{D})$. Then, we performed a pooled analysis of ORs using a random-effects model (including the pooled OR of the relationship between myopia and POAG and the pooled OR of the relationship between myopia and the progression of POAG). We selected the random-effects model because it accounts for both intrastudy and interstudy variability, and we expected significant heterogeneity among the included studies.

Statistical heterogeneity among studies was evaluated using the $I^{2}$ statistic. We performed sensitivity analysis to investigate the contribution of each study to the heterogeneity [38]. Publication bias was evaluated using Egger's test. All statistical analyses were performed with the open-source R program (version 4.0). For all analysis, a 2 -sided $p$ value less than 0.05 was regarded as significant. 
Fig. 1. Flowchart of study selection for analyzing the association between myopia and POAG and the progression of POAG.

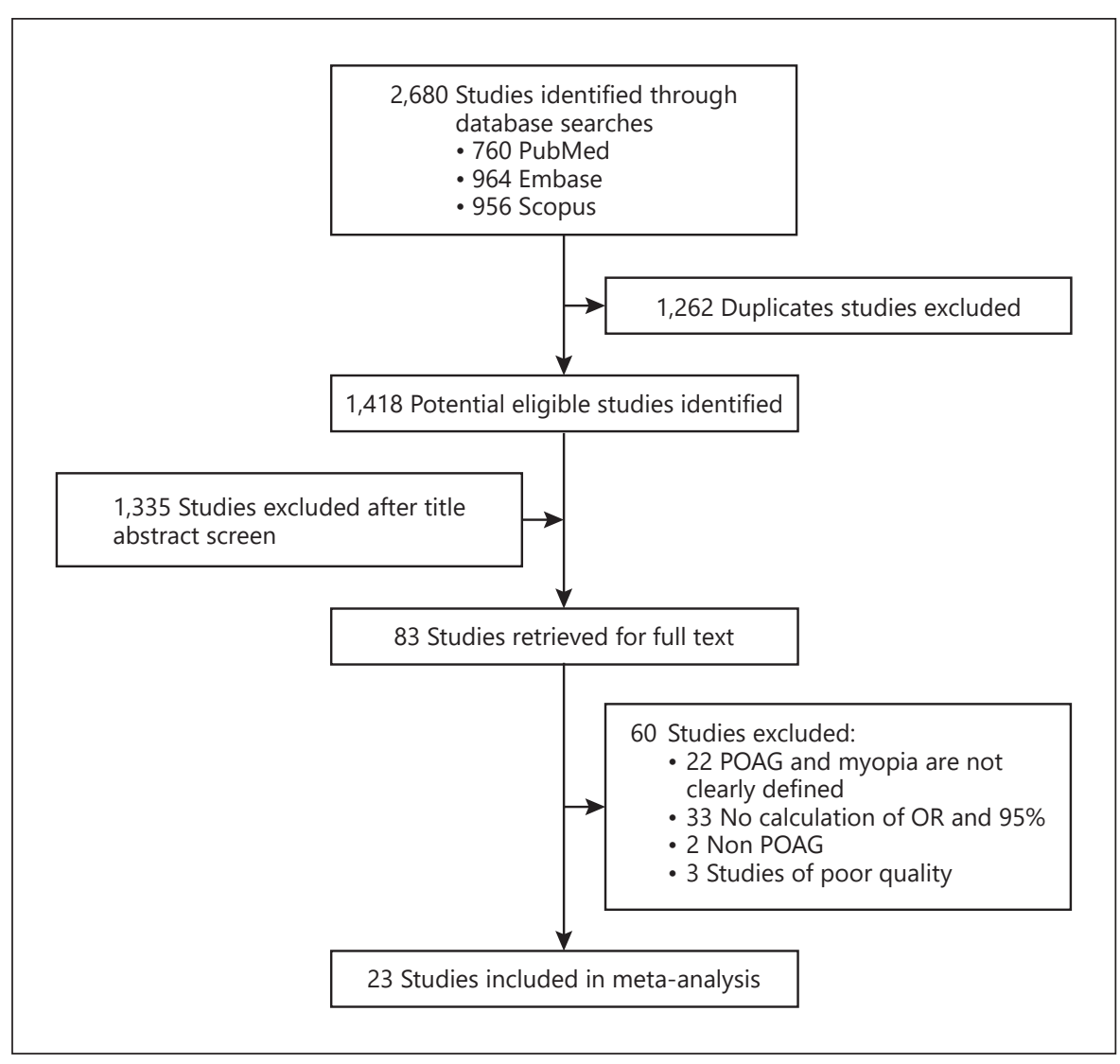

\section{Results}

Figure 1 shows the flowchart of study selection for analyzing the correlation between "myopia and POAG" and "myopia and progression of POAG." The literature search yielded 2,680 articles, with 760 from PubMed, 964 from Embase, and 956 from Scopus. After a thorough review, 1,262 duplicated studies were excluded. Of the 1,418 potential eligible studies identified, 1,335 studies were further excluded after screening the abstract. Full text was retrieved from the remaining 83 studies, 22 of which had no clear definition of POAG or myopia, 33 studies did not calculate the OR value with 95\% CI, 2 studies included a research population consisting of not just POAG patients, and 3 studies were of poor quality. Finally, 60 studies were excluded, and 23 studies were included in this meta-analysis. Of these articles, 16 were cross-sectional studies for "myopia and POAG," and 7 were longitudinal cohort studies for "myopia and progression of POAG." All cross-sectional studies were population-based studies, and all the longitudinal cohort studies were hospitalbased studies.

Myopia and Primary Open-Angle Glaucoma
Tables 1 and 2 summarize the characteristics of the included studies. This meta-analysis comprised a total of 69,249 individuals in cross-sectional studies and 1,687 individuals in cohort studies. The tests for publication bias are shown in the online supplementary material (PRISMA guidelines as online suppl. material 4).

\section{Myopia and POAG}

The 16 studies presented in Table 1 were populationbased cross-sectional studies for "myopia and POAG" $[14,20,25,39-51]$. The classification of myopia was $<-0.5 \mathrm{D}$ in most studies, except for a definition of $<-1.0 \mathrm{D}$ in 5 studies. There were slight differences in the diagnosis of POAG, including glaucomatous visual field (VF) loss and glaucomatous optic-disc changes and the diagnostic criteria from the International Society of Geographic and Epidemiologic Ophthalmology (ISGEO). ORs or the calculation of ORs from multivariable regression analysis were used as the standard of effect evaluation in these studies.

For the analysis of the association between "myopia and POAG," Figure 2 presents the multivariable ORs for 
Table 1. Information abstracted from 16 cross-sectional studies

\begin{tabular}{|c|c|c|c|c|c|c|c|}
\hline Study (design) & Year & Participants & Race & Definition of glaucoma & $\begin{array}{l}\text { Classification of myopia } \\
\text { (SEq in diopters) }\end{array}$ & OR $(95 \% \mathrm{Cl})$ & Adjusted covariates \\
\hline $\begin{array}{l}\text { Nirmalan et al. [12] } \\
\text { (cross-sectional) }\end{array}$ & 2003 & $\begin{array}{l}5,150 \text { (male: } 45.5 \% \text { ) } \\
\text { age: } \geq 40 \mathrm{yr}\end{array}$ & Indian & $\begin{array}{l}\text { GVFL, C/D R } \geq 0.9 \text { or asymmetry } \\
\geq 0.3 \text {, optic-disc abnormalities, } \\
\text { and normal gonioscopy }\end{array}$ & $\begin{array}{l}\text { None }(>-0.5) \\
\text { Mild }(\leq-0.5 \text { to }>-3.0) \\
\text { Moderate }(<-3.1 \text { to }>-6.0) \\
\text { Severe }(\leq-6.0)\end{array}$ & $3.9(1.6,9.5)$ & $\begin{array}{l}\text { Age, gender, } \\
\text { diabetes, } \\
\text { hypertension, and } \\
\text { PXF }\end{array}$ \\
\hline $\begin{array}{l}\text { Liang et al. [19] } \\
\text { (cross-sectional) }\end{array}$ & 2011 & $\begin{array}{l}\text { 6,716 (male: } 46.6 \%) \\
\text { age: } 54.4 \pm 10.8 \mathrm{yr}\end{array}$ & Chinese & Vertical cup/disc ratios and VFs & $\begin{array}{l}\text { None }(>-0.5) \\
\text { Mild }(\leq-0.5 \text { to }>-3.0) \\
\text { Moderate }(<-3.1 \text { to }>-6.0) \\
\text { High }(<-6.1)\end{array}$ & $1.8(0.17,19.3)$ & $\begin{array}{l}\text { Age, axial length, } \\
\text { and IOP }\end{array}$ \\
\hline $\begin{array}{l}\text { Kim et al. [51] } \\
\text { (cross-sectional) }\end{array}$ & 2016 & $\begin{array}{l}\text { 13,831 (male: } \\
43.1 \% \text { ) age: } \\
55.1 \pm 0.2 \mathrm{yr}\end{array}$ & Korean & $\begin{array}{l}\text { Determination of glaucoma } \\
\text { diagnosis from ISGEO }\end{array}$ & $\begin{array}{l}\text { None }(\geq-0.5) \\
\text { Low to moderate }(>-6.0 \text { to }<-0.5) \\
\text { High }(\leq-6.0)\end{array}$ & $3.50(1.96,6.26)$ & $\begin{array}{l}\text { Age, gender, IOP, } \\
\text { hypertension, and } \\
\text { BMI }\end{array}$ \\
\hline $\begin{array}{l}\text { Chen et al. [50] } \\
\text { (cross-sectional) }\end{array}$ & 2016 & $\begin{array}{l}375 \text { (male: } 35.2 \%) \\
\text { age: } 43.7 \pm 11.2 \mathrm{yr}\end{array}$ & Chinese & $\begin{array}{l}\text { RNFL, glaucomatous optic } \\
\text { neuropathy, and VF defect }\end{array}$ & $\begin{array}{l}\text { Excessive }(<-10.0) \\
\text { High }(\geq-10.0 \text { to }<-6.0) \\
\text { Moderate to low }(\geq-6.0 \text { to }<-1.0) \\
\text { None }(>-1.0)\end{array}$ & $3.65(2.28,5.85)$ & $\begin{array}{l}\text { Age, } \mathrm{SE}, \mathrm{IOP}, \text { disc } \\
\text { area, rim area, } \mathrm{C} / \mathrm{D} \\
\mathrm{R} \text {, tilt ratio, and disc } \\
\text { area categories }\end{array}$ \\
\hline $\begin{array}{l}\text { Vijaya et al. [39] } \\
\text { (cross-sectional) }\end{array}$ & 2008 & $\begin{array}{l}3,850 \text { (male: } 44.4 \% \text { ) } \\
\text { age: } 54.8 \pm 10.6 \mathrm{yr}\end{array}$ & Indian & $\begin{array}{l}\text { Determination of glaucoma } \\
\text { diagnosis from ISGEO }\end{array}$ & Myopia $(<-0.5)$ & $0.70(0.40,1.21)$ & $\begin{array}{l}\text { Age, gender, and } \\
\text { IOP }\end{array}$ \\
\hline $\begin{array}{l}\text { Sia et al. [40] } \\
\text { (cross-sectional) }\end{array}$ & 2010 & $\begin{array}{l}\text { 1,375 (male: } 36.1 \%) \\
\text { age: } 57.0 \pm 10.6 \mathrm{yr}\end{array}$ & Sri Lanka & $\begin{array}{l}\text { Determination of glaucoma } \\
\text { diagnosis from ISGEO }\end{array}$ & Myopia $(<-0.5)$ & $4.71(2.13,10.39)$ & $\begin{array}{l}\text { Age, } 1 O P \text {, and axial } \\
\text { length }\end{array}$ \\
\hline $\begin{array}{l}\text { Casson et al. [41] } \\
\text { (cross-sectional) }\end{array}$ & 2007 & $\begin{array}{l}2,481 \text { age: } 56.2 \pm 12 \\
y r\end{array}$ & Australia & $\begin{array}{l}\text { Determination of glaucoma } \\
\text { diagnosis from ISGEO }\end{array}$ & Myopia $(<-0.5)$ & $2.82(1.28,6.23)$ & Age and IOP \\
\hline $\begin{array}{l}\text { Kuzin et al. [16] } \\
\text { (cross-sectional) }\end{array}$ & 2010 & $\begin{array}{l}5,927 \text { (male: } 42 \%) \\
\text { age: } \geq 40 \mathrm{yr}\end{array}$ & American & $\begin{array}{l}\text { Glaucomatous VF abnormality } \\
\text { and/or compatible, } \\
\text { glaucomatous optic-disc } \\
\text { damage in at least } 1 \text { eye }\end{array}$ & Myopia $(<-1)$ & $1.82(1.2,2.77)$ & $\begin{array}{l}\text { Age, gender, IOP, } \\
\text { diabetes, family } \\
\text { history, and } \mathrm{AL}\end{array}$ \\
\hline $\begin{array}{l}\text { Vijaya et al. [20] } \\
\text { (cross-sectional) }\end{array}$ & 2010 & $\begin{array}{l}3,280 \text { (male: } 45.5 \%) \\
\text { age: } 58.2 \pm 10.9\end{array}$ & Singapore & $\begin{array}{l}\text { Determination of glaucoma } \\
\text { diagnosis from ISGEO }\end{array}$ & $\begin{array}{l}\text { Myopia }(<-0.5) \\
\text { Emmetropia }(\geq-0.5 \text { to }<+0.5) \\
\text { Hyperopia }(\geq+0.5)\end{array}$ & $2.87(1.09,7.54)$ & $\begin{array}{l}\text { Age, gender, and } \\
\mathrm{AL}\end{array}$ \\
\hline $\begin{array}{l}\text { Vijaya et al. [43] } \\
\text { (cross-sectional) }\end{array}$ & 2014 & $\begin{array}{l}\text { 4,316 (male: } 44.8 \%) \\
\text { age: } 58.4 \pm 9.7\end{array}$ & Indian & $\begin{array}{l}\text { Determination of glaucoma } \\
\text { diagnosis from ISGEO }\end{array}$ & $\begin{array}{l}\text { Myopia }(<-0.5) \\
\text { Emmetropia }(\geq-0.5 \text { to }<+0.5) \\
\text { Hyperopia }(\geq+0.5)\end{array}$ & $1.7(1.13,2.56)$ & Age and gender \\
\hline $\begin{array}{l}\text { Leske et al. [44] } \\
\text { (cross-sectional) }\end{array}$ & 2001 & $\begin{array}{l}230 \text { (male: } 52 \%) \\
\text { age: } 68 \mathrm{yr}\end{array}$ & American & $\begin{array}{l}\text { Both VF and optic-disc findings } \\
\text { in at least one eye }\end{array}$ & Myopia $(<-0.5)$ & $2.82(1.50,5.30)$ & Age and gender \\
\hline $\begin{array}{l}\text { Mitchell et al. [25] } \\
\text { (cross-sectional) }\end{array}$ & 1999 & 3,654 age: $49-97 \mathrm{yr}$ & Australia & $\begin{array}{l}\text { Optic-disc cupping with rim } \\
\text { thinning (cup-disc ratio USD } 0.7 \\
\text { or cup-disc asymmetry \$ 0.3) } \\
\text { and characteristic VF loss }\end{array}$ & Myopia $(\leq-1)$ & $3.30(1.70,6.40)$ & $\begin{array}{l}\text { Age, gender, and } \\
\text { other known } \\
\text { glaucoma risk } \\
\text { factors }\end{array}$ \\
\hline $\begin{array}{l}\text { Topouzis et al. [45] } \\
\text { (cross-sectional) }\end{array}$ & 2011 & $\begin{array}{l}\text { 2,554 (male: } 60 \% \text { ) } \\
\text { age: } 72.7 \pm 6.1 \mathrm{yr}\end{array}$ & American & $\begin{array}{l}\text { Notching or cup-to-disc ratio } \\
\text { asymmetry of } 0.2 \text { and functional } \\
\text { damage required glaucomatous } \\
\text { VF defect }\end{array}$ & $\begin{array}{l}\text { Mild ( } \geq-3 \text { to }<-1.00) \\
\text { Moderate to high }(<-3.00)\end{array}$ & $\begin{array}{l}1.83(0.95,3.52) \\
2.40(1.24,4.64)\end{array}$ & Age and gender \\
\hline $\begin{array}{l}\text { Tham et al. [14] } \\
\text { (cross-sectional) }\end{array}$ & 2017 & $\begin{array}{l}9,209 \text { (male: } 49 \% \text { ) } \\
\text { age: } 58.2 \pm 10.1 \mathrm{yr}\end{array}$ & Singapore & $\begin{array}{l}\text { Determination of glaucoma } \\
\text { diagnosis from ISGEO }\end{array}$ & Myopia $(<-0.5)$ & $1.07(1.02,1.12)$ & $\begin{array}{l}\text { Age, gender, } \\
\text { ethnicity, and CCT }\end{array}$ \\
\hline $\begin{array}{l}\text { Suzuki Y et al. [23] } \\
\text { (cross-sectional) }\end{array}$ & 2006 & $\begin{array}{l}119 \text { (male: } 47.9 \%) \\
\text { age: } 63.8 \pm 12 \mathrm{yr}\end{array}$ & Japan & $\begin{array}{l}\text { Determination of glaucoma } \\
\text { diagnosis from ISGEO }\end{array}$ & Myopia $(\leq-1)$ & $1.85(1.03,3.32)$ & Age and gender \\
\hline $\begin{array}{l}\text { Pan et al. [47] } \\
\text { (cross-sectional) }\end{array}$ & 2013 & $\begin{array}{l}\text { 3,400 (male: } 49.2 \% \text { ) } \\
\text { age: } 40-84\end{array}$ & Indians & $\begin{array}{l}>21 \mathrm{~mm} \mathrm{Hg}, \mathrm{CDR}>0.6 \text {, } \\
\text { abnormal anterior segment } \\
\text { deposit, occludable anterior } \\
\text { chamber angle, findings } \\
\text { consistent with secondary } \\
\text { glaucoma, and history of } \\
\text { glaucoma }\end{array}$ & Myopia $(<-0.5)$ & $5.90(2.68,12.98)$ & Age and gender \\
\hline
\end{tabular}

GVFL, glaucomatous visual field loss; PXF, pseudoexfoliation; BMI, body mass index; RNFL, retinal nerve-fiber layers; SE, spherical equivalent; C/D R, cup-to-disc ratio. 
Table 2. Information abstracted from 7 cohort studies

\begin{tabular}{|c|c|c|c|c|c|c|c|c|}
\hline Study (design) & Year & $\begin{array}{l}\text { Population (POAG } \\
\text { patients) }\end{array}$ & $\begin{array}{l}\text { Race/ } \\
\text { ethnicity }\end{array}$ & Definition of glaucoma & $\begin{array}{l}\text { Specific indicators for } \\
\text { diagnosing the progress } \\
\text { of POAG }\end{array}$ & $\begin{array}{l}\text { Spherical } \\
\text { equivalent }\end{array}$ & OR $(95 \% \mathrm{CI})$ & Adjusted covariates \\
\hline $\begin{array}{l}\text { Lee et al. [53] } \\
\text { (cohort, } \\
\text { retrospective) }\end{array}$ & 2008 & $\begin{array}{l}262 \text { (male: } 62.2 \% \text { ) } \\
\text { Age: } 48.6 \pm 14.2 \mathrm{yr} \\
\text { Follow-up: } 8.7 \pm 2.2 \\
\text { yr }\end{array}$ & Chinese & $\begin{array}{l}\text { GVFL, optic-disc } \\
\text { abnormalities, } \\
\text { glaucomatous changes } \\
\text { of the optic nerve head, } \\
\text { C/D R }\end{array}$ & Progression of VF loss & $-5.1 \pm 4.2 \mathrm{D}$ & $4.686(2.078,10.568)$ & $\begin{array}{l}\text { Age, CCT, mean IOP, } \\
\text { and VCDR }\end{array}$ \\
\hline $\begin{array}{l}\text { Qiu et al. [30] } \\
\text { (cohort, } \\
\text { retrospective) }\end{array}$ & 2015 & $\begin{array}{l}270 \text { (male: } 60.0 \% \text { ) } \\
\text { Age: } 45.9 \pm 15.3 \mathrm{yr} \\
\text { Follow-up: } 5.6 \pm 2.7 \\
\text { yr }\end{array}$ & Chinese & $\begin{array}{l}\text { Glaucomatous optic } \\
\text { neuropathy with } \\
\text { notching, rim thinning, } \\
\text { RNFL defect, and GVFL }\end{array}$ & $\begin{array}{l}\text { Fast progression: } \mathrm{MD} \\
\text { slope }>0.22 \mathrm{~dB} / \mathrm{yr} \\
\text { Slow progression: } \mathrm{MD} \\
\text { slope } \leq 0.22 \mathrm{~dB} / \mathrm{yr}\end{array}$ & $-3.7 \pm 3.0 \mathrm{D}$ & $\begin{array}{l}\text { Mild myopia: } 0.796 \\
\text { (0.520, 1.218) } \\
\text { Moderate myopia: } \\
0.282(0.193,0.411) \\
\text { Marked myopia: } \\
0.425(0.292,0.620)\end{array}$ & $\begin{array}{l}\text { Age, gender, right/ } \\
\text { left eye, CCT, BCVA, } \\
\text { VCDR, IOP, initial } \\
\text { MD, follow-up } \\
\text { period, SE, and AL }\end{array}$ \\
\hline $\begin{array}{l}\text { Lee et al. [31] } \\
\text { (cohort, } \\
\text { retrospective) }\end{array}$ & 2015 & $\begin{array}{l}369 \text { (male: } 52.0 \% \text { ) } \\
\text { Age: NMG: } \\
65.0 \pm 11.4 \mathrm{yr} \\
\text { MMG: } 60.4 \pm 12.5 \mathrm{yr} \\
\text { HMG: } 49.6 \pm 13.7 \mathrm{yr} \\
\text { Follow-up: } 4.4 \pm 1.0 \\
\text { yr }\end{array}$ & Korean & $\begin{array}{l}\text { Focal thinning of the } \\
\text { neuroretinal rim or } \\
\text { notching, RNFL defect, } \\
\text { and GVFL }\end{array}$ & $\begin{array}{l}\text { Optic disc/RNFL } \\
\text { progression and VF } \\
\text { progression }\end{array}$ & $\begin{array}{l}\text { NMG: } \\
0.71 \pm 1.04 \mathrm{D} \\
\text { MMG: } \\
-1.77 \pm 1.45 \mathrm{D} \\
\text { HMG: } \\
-9.21 \pm 3.57 \mathrm{D}\end{array}$ & $\begin{array}{l}\text { MMG: } 0.830(0.470, \\
1.539) \\
\text { HMG: } 0.868(0.300 \\
2.512)\end{array}$ & $\begin{array}{l}\text { Age, sex, CCT, IOP, } \\
\text { baseline VF MD, } \\
\text { baseline RNFL } \\
\text { thickness, and } \\
\text { baseline GCIPL } \\
\text { thickness }\end{array}$ \\
\hline $\begin{array}{l}\text { Kim et al. [54] } \\
\text { (cohort, } \\
\text { retrospective) }\end{array}$ & 2012 & $\begin{array}{l}313 \text { (male: } 49.2 \%) \\
\text { Age: } R C S: \\
41.5 \pm 11.3 \text { yr } \\
\text { Non-RCS: } \\
56.7 \pm 11.5 \text { yr } \\
\text { Follow-up: } 2.7 \pm 0.4 \\
\text { yr }\end{array}$ & Korean & $\begin{array}{l}\text { Glaucomatous optic-disc } \\
\text { changes, diffuse or focal } \\
\text { neural-rim thinning, disc } \\
\text { hemorrhage or RNFL } \\
\text { defects, and GVFL }\end{array}$ & $\begin{array}{l}\text { Structural progression: } \\
\text { optic disc and red-free } \\
\text { RNFL photographs. } \\
\text { Functional progression: } \\
2 \text { VF methods (EA and } \\
\text { TA) }\end{array}$ & $\begin{array}{l}\text { RCS group: } \\
-0.9 \pm 0.7 \mathrm{D}: \\
\text { Non-RCS } \\
\text { group: } \\
-0.8 \pm 0.7 \mathrm{D}\end{array}$ & $0.936(0.873,1.003)$ & $\begin{array}{l}\text { Baseline age, sex, VF, } \\
\text { AL, any history of } \\
\text { RCS }\end{array}$ \\
\hline $\begin{array}{l}\text { Lee et al. [52] } \\
\text { (cohort, } \\
\text { retrospective) }\end{array}$ & 2015 & $\begin{array}{l}85 \text { (male: } 63.5 \% \text { ) } \\
\text { Age: } 48.3 \pm 13.1 \mathrm{yr} \\
\text { Follow-up: } 4.1 \pm 1.1 \\
\text { yr }\end{array}$ & Korean & $\begin{array}{l}\text { Glaucomatous optic-disc } \\
\text { changes, such as diffuse } \\
\text { or focal neural-rim } \\
\text { thinning, disc } \\
\text { hemorrhage, RNFL } \\
\text { defects, and GVFL }\end{array}$ & $\begin{array}{l}\text { Structural progression: } \\
\text { optic disc and red-free } \\
\text { RNFL photographs. } \\
\text { Functional progression: } \\
\text { VF assessment }\end{array}$ & $-5.5 \pm 3.4 \mathrm{D}$ & $1.006(0.919,1.101)$ & $\begin{array}{l}\text { Age, tilt ratio, } \\
\text { absolute torsional } \\
\text { degree, IOP, CCT, } \\
\text { average RNFL } \\
\text { thickness, AL, disc } \\
\text { hemorrhage, initial } \\
\text { VF MD, and positive } \\
\text { torsional direction }\end{array}$ \\
\hline $\begin{array}{l}\text { Naito et al. } \\
\text { [55] (cohort, } \\
\text { retrospective) }\end{array}$ & 2016 & $\begin{array}{l}156 \text { (male: } 39.7 \% \text { ) } \\
\text { Age: } 64.6 \pm 12.9 \mathrm{yr} \\
\text { Follow-up: } 7.6 \pm 2.0 \\
\text { yr }\end{array}$ & Japanese & $\begin{array}{l}\text { Obvious glaucomatous } \\
\text { VF defects in accordance } \\
\text { with Anderson's criteria }\end{array}$ & $\begin{array}{l}\text { VF progression: a } \\
\text { significantly negative } \\
\text { value of MD slope }(p< \\
0.05) \text { at the final VF test }\end{array}$ & $-2.8 \pm 3.7 \mathrm{D}$ & $1.13(1.03,1.25)$ & $\begin{array}{l}\text { IOP reduction rate } \\
\text { (per } 1 \%)\end{array}$ \\
\hline $\begin{array}{l}\text { Han et al. [56] } \\
\text { (cohort, } \\
\text { retrospective) }\end{array}$ & 2016 & $\begin{array}{l}232 \text { (male: 58.6\%) } \\
\text { Age: nonmyopia: } \\
46.0 \pm 11.4 \mathrm{yr} \\
\text { Myopia: } 45.6 \pm 12.0 \\
\text { yr } \\
\text { Follow-up: } \\
\text { nonmyopia: } \\
10.0 \pm 2.4 \mathrm{yr} \\
\text { Myopia: } 9.8 \pm 2.7 \mathrm{yr}\end{array}$ & Korean & $\begin{array}{l}\text { Glaucomatous optic-disc } \\
\text { changes (VCDR }>0.7 \text { ), } \\
\text { diffuse or focal neural- } \\
\text { rim thinning, disc } \\
\text { hemorrhage, RNFL } \\
\text { defects, and GVFL }\end{array}$ & $\begin{array}{l}\text { Glaucoma progression } \\
\text { analysis (GPA; Carl Zeiss } \\
\text { Meditec Inc.)software, } \\
\text { which supplies both EA } \\
\text { and TA }\end{array}$ & $\begin{array}{l}\text { Nonmyopia: } \\
-1.2 \pm 2.3 \mathrm{D} \\
\text { myopia: } \\
-4.5 \pm 2.7 \mathrm{D}\end{array}$ & $0.955(0.900,1.014)$ & $\begin{array}{l}\text { Age, CCT, AL, IOP, } \\
\text { number of } \\
\text { medications, } \\
\text { baseline MD, disc } \\
\text { ovality index, tilt } \\
\text { direction, and PPA- } \\
\text { to-disc ratio }\end{array}$ \\
\hline
\end{tabular}

GVFL, glaucomatous visual field loss; CCT, central corneal thickness; VCDR, vertical cup-to-disc ratio; RNFL, retinal nerve-fiber layers; BCVA, best-corrected visual acuity; SE, spherical equivalent; GCIPL, ganglion-cell inner plexiform complex; RCS, refractive corneal surgery; EA and TA, event-based and trend-based analysis; PPA, peripapillary atrophy; C/D R, cup-to-disc ratio.

each study separately and the 16 cross-sectional studies together [14, 20, 25, 39-51]. The pooled OR of the association between myopia and POAG was 2.26 (95\% CI, $1.77-2.89, p<0.001)$ in the random-effects model, indicating myopia was shown to be a risk factor for POAG. There was statistically significant heterogeneity among the cross-sectional studies $\left(I^{2}=86 \% ; p<0.01\right)$.

As shown in Figure 3, we further stratified our analysis according to the different degrees of myopia for the stud- ies that included such stratification. The studies were classified into 3 subgroups: low myopia, moderate myopia, and high myopia. For each subgroup, the pooled ORs were 1.63 (95\% CI: 1.35-1.96), 2.27 (95\% CI: 1.38-3.73), and 3.47 (95\% CI: 2.48-4.85), respectively, all of which were statistically significant, and the conclusion remains the same. 


\begin{tabular}{|c|c|c|c|c|c|c|}
\hline Study & TE SeTE & Odds Ratio & OR & $95 \%-\mathrm{Cl}$ & $\begin{array}{c}\text { Weight } \\
\text { (fixed) }\end{array}$ & $\begin{array}{r}\text { Weight } \\
\text { (random) }\end{array}$ \\
\hline Lingam Vijaya 2008 (Rural)[29] & -0.360 .2803 & tí & 0.70 & {$[0.40 ; 1.21]$} & $0.6 \%$ & $4.9 \%$ \\
\hline Lingam Vijaya 2008 (Urban)[29] & 0.340 .1970 & I & 1.40 & {$[0.95 ; 2.06]$} & $1.3 \%$ & $5.6 \%$ \\
\hline Sia DI 2010[40] & 1.550 .4041 & $D_{i}$ & 4.71 & {$[2.13 ; 10.39]$} & $0.3 \%$ & $3.9 \%$ \\
\hline Robert J Casson 2007[41] & 1.040 .4045 & & 2.82 & {$[1.28 ; 6.23]$} & $0.3 \%$ & $3.9 \%$ \\
\hline Aaron A Kuzin 2010[16] & 0.600 .2134 & $i$ & 1.82 & {$[1.20 ; 2.77]$} & $1.1 \%$ & $5.5 \%$ \\
\hline Perera SA 2010[20] & 1.050 .4930 & 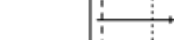 & 2.87 & {$[1.09 ; 7.54]$} & $0.2 \%$ & $3.2 \%$ \\
\hline Lingam Vijaya 2014[43] & 0.530 .2094 & & 1.70 & {$[1.13 ; 2.56]$} & $1.1 \%$ & $5.5 \%$ \\
\hline Leske MC 2001[44] & 1.040 .3220 & & 2.82 & {$[1.50 ; 5.30]$} & $0.5 \%$ & $4.5 \%$ \\
\hline Mitchell P 1999[25] & 1.190 .3382 & ! & 3.30 & {$[1.70 ; 6.40]$} & $0.4 \%$ & $4.4 \%$ \\
\hline Topouzis F 2011 (Low myopia)[45] & 0.600 .3334 & 4 & 1.83 & {$[0.95 ; 3.52]$} & $0.4 \%$ & $4.4 \%$ \\
\hline Topouzis F 2011 (Moderate to high myopia)[45] & 0.880 .3366 & L & 2.40 & {$[1.24 ; 4.64]$} & $0.4 \%$ & $4.4 \%$ \\
\hline Yih-Chung Tham 2017[14] & 0.070 .0239 & 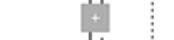 & 1.07 & {$[1.02 ; 1.12]$} & $85.5 \%$ & $6.5 \%$ \\
\hline Yasuyuki Suzuki 2006[23] & 0.620 .2978 & 7 & 1.85 & {$[1.03 ; 3.32]$} & $0.5 \%$ & $4.7 \%$ \\
\hline Chen-Wei Pan 2013[47] & 1.770 .4023 & ' & 5.90 & {$[2.68 ; 12.98]$} & $0.3 \%$ & $3.9 \%$ \\
\hline R Ramakrishnan 2003 (Low myopia)[12] & 1.360 .4544 & ; & 3.90 & {$[1.60 ; 9.50]$} & $0.2 \%$ & $3.5 \%$ \\
\hline R Ramakrishnan 2003 (Moderate myopia))[12] & 0.740 .3893 & I. & 2.10 & {$[0.98 ; 4.50]$} & $0.3 \%$ & $4.0 \%$ \\
\hline R Ramakrishnan 2003 (High myopia))[12] & 1.060 .4258 & & 2.90 & {$[1.26 ; 6.68]$} & $0.3 \%$ & $3.7 \%$ \\
\hline Yuan Bo Liang 2011[19] & 1.550 .5441 & i: & 4.70 & {$[1.62 ; 13.65]$} & $0.2 \%$ & $2.9 \%$ \\
\hline Chen LW 2016 (Low to moderate myopia)[50] & 0.830 .3475 & 17 & 2.29 & {$[1.16 ; 4.53]$} & $0.4 \%$ & $4.3 \%$ \\
\hline Chen LW 2016 (High myopia)[50] & 1.290 .2404 & : & 3.65 & {$[2.28 ; 5.85]$} & $0.8 \%$ & $5.2 \%$ \\
\hline Chen LW 2016 (Low to moderate myopia)[50] & 0.390 .1077 & in & 1.48 & {$[1.20 ; 1.83]$} & $4.2 \%$ & $6.2 \%$ \\
\hline Chen LW 2016 (High myopia)[50] & 1.250 .2962 & & 3.50 & {$[1.96 ; 6.25]$} & $0.6 \%$ & $4.8 \%$ \\
\hline Fixed effect model & & i & 1.17 & {$[1.12 ; 1.22]$} & $100.0 \%$ & -- \\
\hline Random effects model & & & 2.26 & {$[1.77 ; 2.89]$} & -- & $100.0 \%$ \\
\hline Heterogeneity: $I^{2}=86 \%, \tau^{2}=0.2406, p<0.01$ & & $T$ & & & & \\
\hline
\end{tabular}

Fig. 2. Forest plot of risk estimates of the association between myopia and POAG in cross-sectional studies.

\section{Myopia and the Progression of POAG}

The 7 studies presented in Table 2 were longitudinal cohort studies for "myopia and progression of POAG" [32, $33,52-56]$. The mean follow-up period ranged from 2.7 years to 10 years, and the mean spherical equivalent was different. The diagnosis of POAG included VF loss and optic-disc changes. For the diagnosis of POAG progression, the progression of VF loss or structural changes of the optic disc $[32,53,55]$ were considered $[33,52,54,56]$. The OR value or the calculation of the OR value was used as the standard effect evaluation in these studies. Univariable [33, 52] or multivariable regression analyses were performed $[32,53-56]$ in previous studies. For the analysis of the association between "myopia and the progression of POAG," Figure 4 presents the multivariable ORs for each study separately and the 7 cross-sectional studies together [32, 33, $52-56]$. The pooled OR of the association between myopia and the progression of POAG was 0.85 (95\% CI, 0.73-0.99, $p=0.042)$ in the random-effects model, implying myopia may have a protective effect on the progression of POAG. There was statistically significant heterogeneity among the 7 longitudinal cohort studies $\left(I^{2}=90 \% ; p<0.01\right)$.
We classified the different degrees of myopia for the studies into 3 subgroups: low myopia, moderate myopia, and high myopia. For each subgroup, we stratified our analysis to include such stratification, and the pooled OR was statistically significant: the pooled ORs for the mild, moderate, and high myopia subgroups were 0.80 (95\% CI: 0.52-1.22), 0.47 (95\% CI: $0.16-1.36)$, and 0.51 (95\% CI: 0.27-0.94), respectively, as shown in Figure 5.

\section{Discussion}

This meta-analysis was designed for the analysis of the association between "myopia and POAG," and the association between "myopia and the progression of POAG," respectively. The pooled ORs implied that myopia may be a risk factor for POAG but a protective factor for the progression of POAG. This is the first meta-analysis to show that myopia may have protective effects on the progression of POAG, which may provide new insight into the pathogenesis of POAG development. 


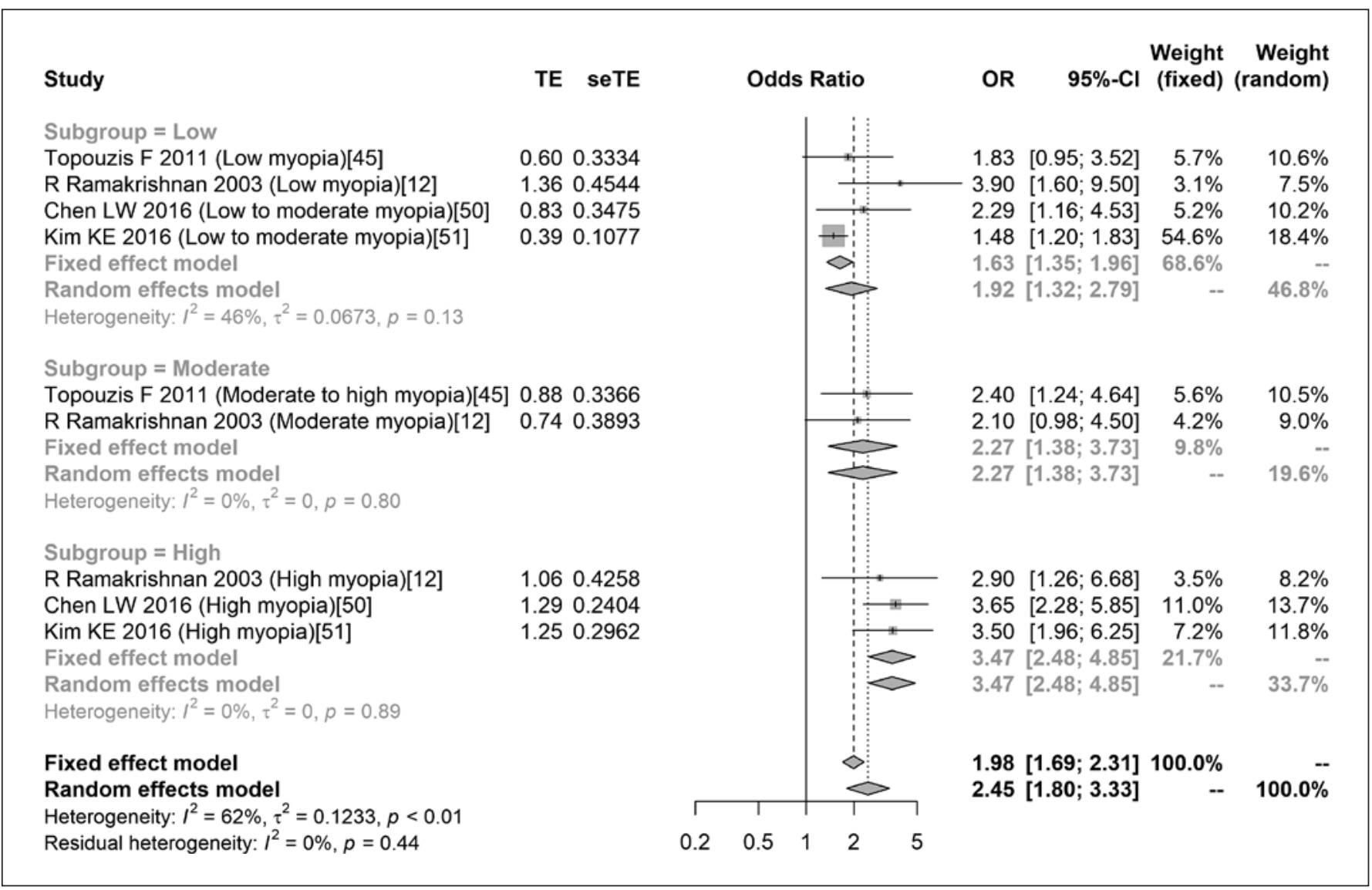

Fig. 3. Subgroup forest plot of risk estimates of the association between myopia and POAG in cross-sectional studies.

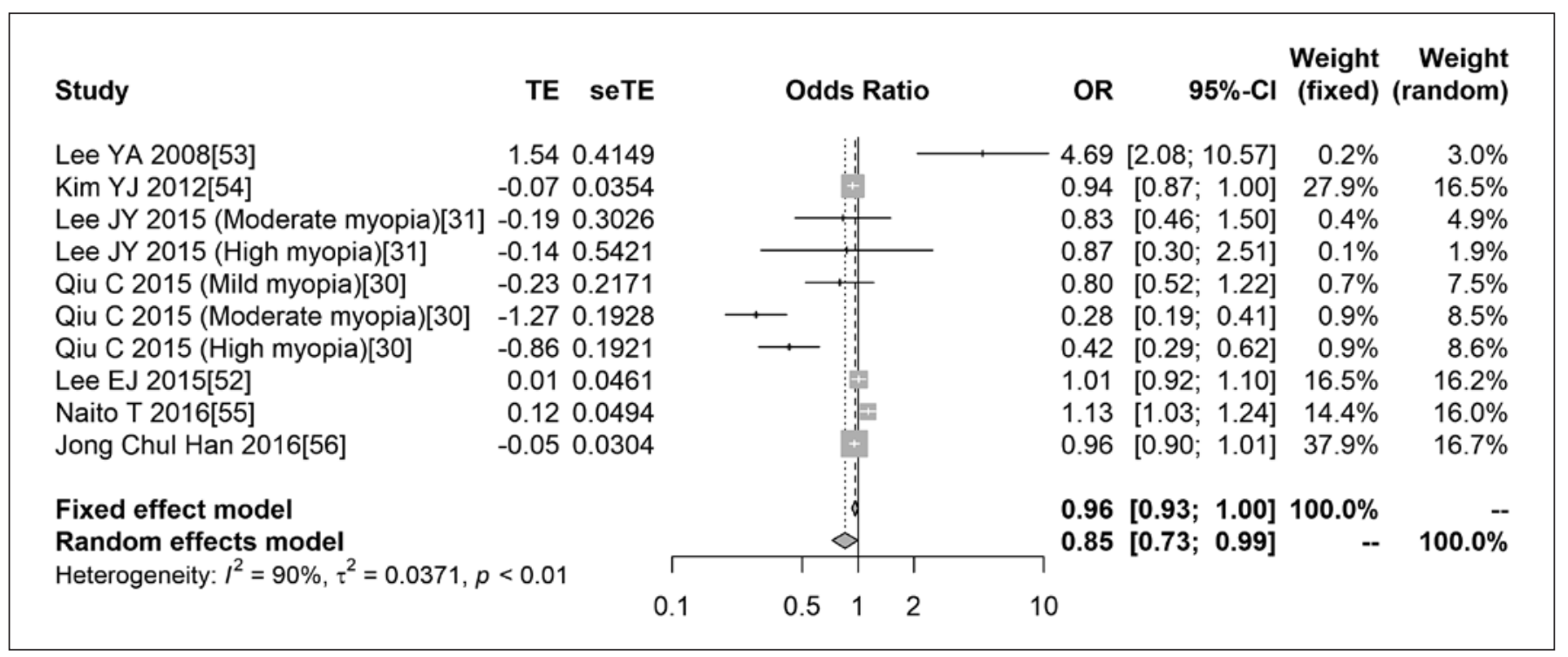

Fig. 4. Forest plot of risk estimates of the association between myopia and POAG in the longitudinal cohort studies. 
Study

Subgroup $=$ Low

Qiu C 2015 (Low myopia)[30]

Fixed effect model

Random effects model

Heterogeneity: not applicable

Subgroup $=$ Moderate

Lee JY 2015 (Moderate myopia)[31] -0.190 .3026$

Qiu C 2015 (Moderate myopia)[30]

Fixed effect model

Random effects model

Heterogeneity: $l^{2}=89 \%, \tau^{2}=0.5183, p<0.01$

Subgroup $=$ High

Lee JY 2015 (High myopia)[31]

Qiu C 2015 (High myopia)[30]

$\begin{array}{ll}-0.14 & 0.5421\end{array}$

Fixed effect model

Random effects model

Heterogeneity: $I^{2}=37 \%, \tau^{2}=0.0978, p=0.21$

\section{Fixed effect model}

Random effects model

Heterogeneity: $I^{2}=78 \%, \tau^{2}=0.2103, p<0.01$

Residual heterogeneity: $I^{2}=81 \%, p<0.01$

TE seTE

$-0.230 .2171$

$-0.870 .1938$
Odds Ratio

OR

(fixed) (random)
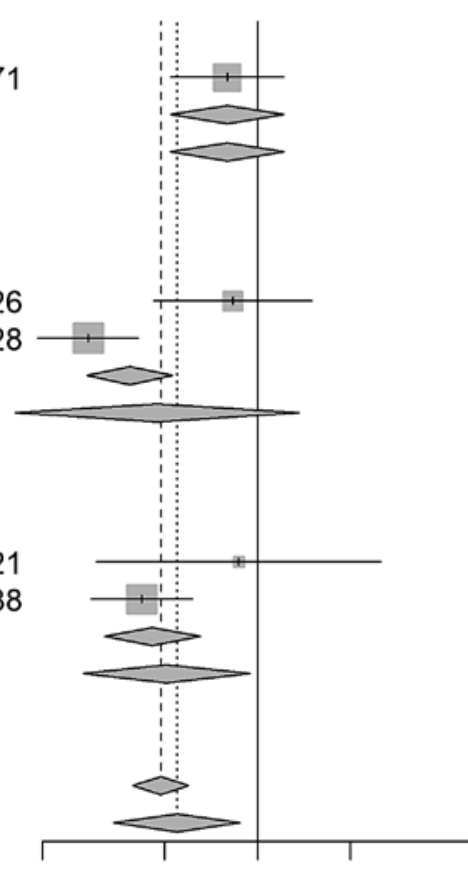

$0.83[0.46 ; 1.50] \quad 12.3 \%$

0.28 [0.19;0.41] $30.2 \%$

$0.39[0.28 ; 0.53] \quad 42.5 \%$

$0.47[0.16 ; 1.36]$

$19.2 \%$

$23.4 \%$

$42.6 \%$

$0.87[0.30 ; 2.51] \quad 3.8 \%$

$0.42[0.29 ; 0.61] 29.9 \%$

$0.46[0.32 ; 0.65] \quad 33.7 \%$

$0.51[0.27 ; 0.94] \quad--$

$11.5 \%$

$23.4 \%$

$34.9 \%$

$0.48[0.39 ; 0.60] 100.0 \%$

$0.55[0.34 ; 0.88] \quad--100.0 \%$

Fig. 5. Subgroup forest plot of risk estimates of the association between myopia and POAG in the longitudinal cohort studies.

We analyzed the cross-sectional studies and longitudinal cohort studies separately. The sample of the cross-sectional studies came from the general population, which covers a wide range and involves a large number of people; thus, the results of the study are suitable for popularization. Moreover, intrinsic selection and information biases are unavoidable. In cohort studies, the chronological order of etiology and disease is definite, and there is no memory bias. Previous studies reported that myopia may be a risk factor for POAG. This study confirmed that myopia is a risk factor for POAG. The possible mechanisms may be as follows: first, the function of the trabecular meshwork could be damaged in myopia, and thus, the outflow resistance of aqueous humor is increased, causing increased IOP. Second, the same IOP may put higher pressure on the head of the optic nerve in myopic eyes. This pressure causes neural tissue strain and results in loss of ganglion cells despite apparently normalized IOP [28]. Therefore, myopic patients may have a greater susceptibility to POAG.

In terms of the relationship between myopia and POAG progression, our results indicate that myopia may be a slightly protective factor for the progression of POAG. This finding is inconsistent with the results of previous articles, especially in high-myopia studies. In the study conducted by Lee et al. [53], the OR for the relationship between myopia and progression of POAG was 4.686 (95\% CI, 2.078-10.568), and the authors observed that patients with POAG and myopia had a greater progression of VF loss. Chihara et al. [57] also proposed that severe myopia, but not mild myopia, was a significant risk factor for VF loss in patients with POAG. In the studies by Lee et al. [53] and Chihara et al. [57], progression of VF loss was taken as the only evaluation criterion for POAG progression; nevertheless, there are other criteria for the progression of POAG. Structural changes, such as elongated AL, tilted optic disc, parapapillary atrophy, and thinning of the lamina cribrosa and parapapillary sclera, can cause VF defects in highly myopic eyes [55]; thus, these changes may aggravate POAG in patients with high myopia.

However, our study found that myopia may be a slightly protective factor on the progression of POAG. One cause of the difference in results between studies might be 
that the progression of VF loss in patients with high myopia was confounded, which inevitably led to the overdiagnosis of POAG progression in previous studies. This problem has been mentioned and corrected in the subsequent articles [32, 33, 52, 54-56]. In a study from Japan, Nitta et al. [27] proposed that highly myopic POAG should be regarded as a combination of myopic optic neuropathy and glaucomatous optic neuropathy. Thus, high myopia and POAG may be 2 risk factors that interact with each other and cause optic neuropathy. Moreover, some other diseases can be complications of high myopia [58]; therefore, the effects of myopia on POAG may be due to secondary complications in some studies. To conclude, there are divergent opinions on the inconsistent conclusions in the current studies, and future research is needed to clarify this issue.

Furthermore, the results of the cross-sectional and longitudinal cohort studies were inconsistent, indicating that there may be some factors of myopia that result in avoidance of POAG progression. In the follow-up of the previous studies, IOP, age, and other factors differed, and these data might have an effect on the relationship between POAG and myopia. In cross-sectional studies, we selected the ORs from the multivariable regression analysis of each study and then performed a pooled analysis, in which case, the effects of these factors could be partially excluded. However, in the cohort studies, because there was no statistical difference between the results of some articles on the relationship between myopia and POAG progression, multivariable regression analysis was not carried out in these studies $[33,52]$.

In addition, a study on the relationship between myopia and nonprogressive glaucoma VF loss found that the presence of lamina cribrosa defect was significantly associated with nonprogressive VF loss [59], suggesting that the negative results of these longitudinal studies may be due to a lamina cribrosa defect. Glaucoma is characterized by elevated IOP, which leads to a difference of in pressure before and after the lamina cribrosa (i.e., between the vitreous cavity and subarachnoid space), forming the trans-lamina cribrosa pressure gradient. Lamina cribrosa defects were formed by stretching the optic disc via an elongation of the AL and were also related to the optic-disc tilt of the aggravating myopic eyeball. While the LC defects formed, the pressure may release [60-63], and the progression of VF loss might thus be slowed down in the eyes. The findings of this study suggest that the release of the trans-lamina cribrosa pressure difference induced by the lamina cribrosa defect may be the cause of the stagnation in VF loss. Hence, myopia may be a slightly protective factor for the progression of POAG. However, these hypotheses should be verified in a prospective study.

This meta-analysis has some limitations. First, different diagnostic criteria for POAG were used in both crosssectional and follow-up studies, but most studies adopted the diagnostic criteria proposed by the ISGEO or similar to that of the ISGEO. Second, there was a slight difference in the degrees of myopia selected in each study. Fortunately, the gap was small and might not have remarkably affected the results. Third, there are very few reports elucidating the relationship between the different degrees of myopia and POAG progression, and more studies are warranted in the future. Moreover, studies used different covariables within their models, which may lead to related bias. Thus, we have added the different degrees of myopia subgroup to further stratify the association. Finally, major published studies might have publication bias, in which, studies that report statistically significant results are more likely to get published than studies that report negative results, leading to our meta-analysis with closer to significant results (online suppl. material 2, 3).

In conclusion, findings from this meta-analysis indicated that myopia may be a risk factor for POAG but also a protective factor for the progression of POAG. Clinicians should be aware of the potential role of myopia in the pathogenesis of glaucoma. Future research should focus on the specific mechanisms underlying the myopiainduced reduction in POAG progression.

\section{Statement of Ethics}

All analyses were based on published studies, and thus, no ethical approval and informed consent are required. Nonetheless, the study adhered fully to the Declaration of Helsinki.

\section{Conflict of Interest Statement}

The authors declare that they have no competing interests.

\section{Funding Sources}

The study is supported by the National Natural Science Foundation of China (GZR-2012-009) and Beijing Traditional Chinese Medicine Technology Development Fund Project (JJ2018-50). 


\section{Author Contributions}

Ningli Wang contributed to the study design and concept; Jian $\mathrm{Wu}$, Jie Hao, and Runzhou Sun contributed to database search; Jian Wu, Jie Hao, and Runzhou Sun contributed to data extracting; Kai Cao contributed to data analysis; JianWu, Jie Hao, and Yifan Du contributed to manuscript writing; Caixia Lin, Yuan Xie, and Ningli Wang contributed to manuscript revising.

\section{Data Availability Statement}

The data of the current study are available from the corresponding author on reasonable request.

\section{References}

1 Wu PC, Huang HM, Yu HJ, Fang PC, Chen CT. Epidemiology of myopia. Asia Pac J Ophthalmol. 2016;5:386-93.

2 Holden BA, Mariotti SP, Kocur I, Resnikoff S, He M. The impact of myopia and high myopia. Paper presented at the World Health Organization-Brien Holden Vision Institute. Sydney: Global Scientific Meeting on Myopia; 2017.

3 Angle J, Wissmann DA. The epidemiology of myopia. Am J Epidemiol. 1980;111(2):220-8.

4 Edwards MH, Lam CS. The epidemiology of myopia in Hong Kong. Ann Acad Med Singap. 2004;33(1):34-8.

5 Saw SM, Katz J, Schein OD, Chew SJ, Chan TK. Epidemiology of myopia. Epidemiol Rev. 1996;18(2):175-87.

6 Juronen E, Tasa G, Veromann S, Parts L, Tiidla A, Pulges R, et al. Polymorphic glutathione S-transferase M1 is a risk factor of primary open-angle glaucoma among Estonians. Exp Eye Res. 2000;71(5):447-52.

7 Quigley HA, Broman AT. The number of people with glaucoma worldwide in 2010 and 2020. Br J Ophthalmol. 2006;90(3):262-7.

8 Marcus MW, de Vries MM, Junoy Montolio FG, Jansonius NM. Myopia as a risk factor for open-angle glaucoma: a systematic review and meta-analysis. Ophthalmology. 2011; 118(10):1989-e2.

9 Knapp A. Glaucoma in Myopic Eyes. Trans Am Ophthalmol Soc. 1925;23:61-70.

10 Coleman AL, Miglior S. Risk factors for glaucoma onset and progression. Surv Ophthalmol. 2008;53(1):S3-10.

11 Qiu M, Wang SY, Singh K, Lin SC. Association between myopia and glaucoma in the United States population. Invest Ophthalmol Vis Sci. 2013;54(1):830-5.

12 Nirmalan PK, Katz J, Robin AL, Tielsch JM, Namperumalsamy P, Kim R, et al. Prevalence of vitreoretinal disorders in a rural population of southern India: the Aravind comprehensive eye study. Arch Ophthalmol. 2004; 122(4):581-6

$13 \mathrm{Xu} \mathrm{L}$, Wang Y, Wang S, Wang Y, Jonas JB High myopia and glaucoma susceptibility the Beijing eye study. Ophthalmology. 2007; 114(2):216-20.

14 Tham YC, Aung T, Fan Q, Saw SM, Siantar RG, Wong TY, et al. Joint effects of intraocular pressure and myopia on risk of primary open-angle glaucoma: the Singapore epide- miology of eye diseases study. Sci Rep. 2016; 6:19320

15 Chon B, Qiu M, Lin SC. Myopia and glauco$\mathrm{ma}$ in the South Korean population. Invest Ophthalmol Vis Sci. 2013;54(10):6570-7.

16 Kuzin AA, Varma R, Reddy HS, Torres M, Azen SP; Los Angeles Latino Eye Study Group. Ocular biometry and open-angle glaucoma: the Los Angeles Latino eye study. Ophthalmology. 2010;117(9):1713-9.

17 Weih LM, Nanjan M, McCarty CA, Taylor HR. Prevalence and predictors of open-angle glaucoma: results from the visual impairment project. Ophthalmology. 2001;108(11):196672.

18 Garudadri C, Senthil S, Khanna RC, Sannapaneni K, Rao HB. Prevalence and risk factors for primary glaucomas in adult urban and rural populations in the Andhra Pradesh eye disease study. Ophthalmology. 2010;117(7): 1352-9.

19 Liang YB, Friedman DS, Zhou Q, Yang X, Sun LP, Guo LX, et al. Prevalence of primary open angle glaucoma in a rural adult Chinese population: the Handan eye study. Invest Ophthalmol Vis Sci. 2011;52(11):8250-7.

20 Vijaya SA, Wong TY, Tay WT, Foster PJ, Saw SM, Aung T. Refractive error, axial dimensions, and primary open-angle glaucoma: the Singapore Malay Eye Study. Archive Ophthalmol. 2010;128(7):900-5.

$21 \mathrm{Wu}$ SY, Nemesure B, Leske MC. Refractive errors in a black adult population: the Barbados eye study. Invest Ophthalmol Vis Sci. 1999; 40(10):2179-84.

22 Wong TY, Klein BE, Klein R, Knudtson M, Lee KE. Refractive errors, intraocular pressure, and glaucoma in a white population. Ophthalmology. 2003;110(1):211-7.

23 Suzuki Y, Iwase A, Araie M, Yamamoto T, Abe H, Shirato S, et al. Risk factors for openangle glaucoma in a Japanese population: the Tajimi Study. Ophthalmology. 2006;113(9): 1613-7.

24 Shim SH, Sung KR, Kim JM, Kim HT, Jeong J, Kim CY, et al. The prevalence of open-angle glaucoma by age in myopia: the Korea national health and nutrition examination survey. Curr Eye Res. 2017;42(1):65-71.

25 Mitchell P, Hourihan F, Sandbach J, Wang JJ. The relationship between glaucoma and myopia: the blue mountains eye study. Ophthalmology. 1999;106(10):2010-5.
26 Song MK, Sung KR, Han S, Lee JE, Yoon JY, Park JM, et al. Progression of primary open angle glaucoma in asymmetrically myopic eyes. Graefes Arch Clin Exp Ophthalmol. 2016;254(7):1331-7.

27 Nitta K, Sugiyama K, Wajima R, Tachibana G. Is high myopia a risk factor for visual field progression or disk hemorrhage in primary open-angle glaucoma? Clin Ophthalmol. 2017;11:599-604.

28 Park HY, Hong KE, Park CK. Impact of age and myopia on the rate of visual field progression in glaucoma patients. Medicine. 2016; 95(21):e3500.

29 Yang Y, Li Z, Wang N, Wu L, Zhen Y, Wang $\mathrm{T}$, et al. Intraocular pressure fluctuation in patients with primary open-angle glaucoma combined with high myopia. J Glaucoma. 2014;23(1):19-22.

30 Nowroozzadeh M. Relation of glaucoma progression and myopia: the role of axial length. Eur J Ophthalmol. 2009;19(2):327.

31 Jonas JB, Dichtl A. Optic disc morphology in myopic primary open-angle glaucoma. Graefes Arch Clin Exp Ophthalmol. 1997;235(10): 627-33.

32 Qiu C, Qian S, Sun X, Zhou C, Meng F. Axial myopia is associated with visual field prognosis of primary open-angle glaucoma. PLoS One. 2015;10(7):e0133189.

33 Lee JY, Sung KR, Han S, Na JH. Effect of myopia on the progression of primary open-angle glaucoma. Invest Ophthalmol Vis Sci. 2015;56(3):1775-81.

34 Yoshino T, Fukuchi T, Togano T, Sakaue Y, Seki M, Tanaka T, et al. Rate of progression of total, upper, and lower visual field defects in patients with open-angle glaucoma and high myopia. Jpn J Ophthalmol. 2016;60(2):78-85.

35 Miki A, Ikuno Y, Asai T, Usui S, Nishida K. Defects of the lamina cribrosa in high myopia and glaucoma. PloS One. 2015;10(9):e0137909.

36 Jonas JB, Berenshtein E, Holbach L. Lamina cribrosa thickness and spatial relationships between intraocular space and cerebrospinal fluid space in highly myopic eyes. Invest Ophthalmol Vis Sci. 2004;45(8):2660-5.

37 Stroup DF, Berlin JA, Morton SC, Olkin I, Williamson GD, Rennie D, et al. Meta-analysis of observational studies in epidemiology: a proposal for reporting. Meta-analysis of observational studies in epidemiology (MOOSE) group. JAMA. 2000;283(15):2008-12. 
38 Egger M, Smith GD, Phillips AN. Meta-analysis: principles and procedures. BMJ. 1997; 315(7121):1533-7.

39 Vijaya L, George R, Baskaran M, Arvind H, Raju P, Ramesh SV, et al. Prevalence of primary open-angle glaucoma in an urban south Indian population and comparison with a rural population. The chennai glaucoma study. Ophthalmology. 2008;115(4):648-e1.

40 Sia DI, Edussuriya K, Sennanayake S, Senaratne T, Selva D, Casson RJ. Prevalence of and risk factors for primary open-angle glaucoma in central Sri Lanka: the kandy eye study. Ophthalmic Epidemiol. 2010;17(4):211-6.

41 Casson RJ, Gupta A, Newland HS, McGovern S, Muecke J, Selva D, et al. Risk factors for primary open-angle glaucoma in a Burmese population: the Meiktila eye study. Clin Exp Ophthalmol. 2007;35(8):739-44.

42 Kuzin AA, Varma R, Reddy HS, Torres M, Azen SP. Ocular biometry and open-angle glaucoma: the Los Angeles latino eye study. Ophthalmology. 2010;117(9):1713-9.

43 Vijaya L, Rashima A, Panday M, Choudhari NS, Ramesh SV, Lokapavani V, et al. Predictors for incidence of primary open-angle glaucoma in a South Indian population: the Chennai eye disease incidence study. Ophthalmology. 2014;121(7):1370-6.

44 Leske MC, Nemesure B, He Q, Wu SY, Fielding Hejtmancik J, Hennis A. Patterns of openangle glaucoma in the Barbados family study. Ophthalmology. 2001;108(6):1015-22.

45 Topouzis F, Wilson MR, Harris A, Founti P, $\mathrm{Yu} F$, Anastasopoulos E, et al. Risk factors for primary open-angle glaucoma and pseudoexfoliative glaucoma in the Thessaloniki eye study. Am J Ophthalmol. 2011;152(2):219-e1.

46 Suzuki Y, Iwase A, Araie M, Yamamoto T, Abe H, Shirato S, et al. Risk factors for openangle glaucoma in a Japanese population: the
Tajimi Study. Ophthalmology. 2006;113(9): 1613-7.

47 Pan CW, Cheung CY, Aung T, Cheung CM, Zheng YF, Wu RY, et al. Differential associations of myopia with major age-related eye diseases: the Singapore Indian eye study. Ophthalmology. 2013;120(2):284-91.

48 Ramakrishnan R, Nirmalan PK, Krishnadas R, Thulasiraj RD, Tielsch JM, Katz J, et al. Glaucoma in a rural population of southern India: the Aravind comprehensive eye survey. Ophthalmology. 2003;110(8):1484-90.

49 Liang YB, Friedman DS, Zhou Q, Yang X, Sun LP, Guo LX, et al. Prevalence of primary open angle glaucoma in a rural adult Chinese population: the Handan eye study. Invest Ophthalmol Vis Sci. 2011;52(11):8250-7.

50 Chen LW, Lan YW, Hsieh JW. The optic nerve head in primary open-angle glaucoma eyes with high myopia: characteristics and association with visual field defects. J Glaucoma. 2016;25(6):e569-75.

51 Kim KE, Kim MJ, Park KH, Jeoung JW, Kim $\mathrm{SH}$, Kim CY, et al. Prevalence, awareness, and risk factors of primary open-angle glaucoma: korea national health and nutrition examination survey 2008-2011. Ophthalmology. 2016;123(3):532-41

52 Lee JE, Sung KR, Lee JY, Park JM. Implications of optic disc tilt in the progression of primary open-angle glaucoma. Invest Ophthalmol Vis Sci. 2015;56(11):6925-31.

53 Lee YA, Shih YF, Lin LL, Huang JY, Wang TH. Association between high myopia and progression of visual field loss in primary open-angle glaucoma. J Formos Med Assoc. 2008;107(12):952-7.

54 Kim YJ, Yun SC, Na JH, Tchah HW, Jung JJ, Sung KR. Glaucoma progression in eyes with a history of refractive corneal surgery. Invest Ophthalmol Vis Sci. 2012;53(8):4485-9.
55 Naito T, Yoshikawa K, Mizoue S, Nanno M, Kimura T, Suzumura H, et al. Relationship between visual field progression and baseline refraction in primary open-angle glaucoma. Clin Ophthalmol. 2016;10:1397-403.

56 Han JC, Lee EJ, Kim SH, Kee C. Visual field progression pattern associated with optic disc tilt morphology in myopic open-angle glaucoma. Am J Ophthalmol. 2016;169:33-45.

57 Chihara E, Liu X, Dong J, Takashima Y, Akimoto $M$, Hangai $M$, et al. Severe myopia as a risk factor for progressive visual field loss in primary open-angle glaucoma. Ophthalmologica. 1997;211(2):66-71.

58 Wilson A, Woo G. A review of the prevalence and causes of myopia. Singapore Med J. 1989; 30(5):479-84.

59 Sawada Y, Araie M, Kasuga H, Ishikawa M, Iwata T, Murata K, et al. Focal lamina cribrosa defect in myopic eyes with nonprogressive glaucomatous visual field defect. Am J Ophthalmol. 2018;190:34-49.

60 Kimura Y, Akagi T, Hangai M, Takayama K, Hasegawa T, Suda K, et al. Lamina cribrosa defects and optic disc morphology in primary open angle glaucoma with high myopia. PLoS One. 2014;9(12):e115313.

61 Park HY, Hwang YS, Park CK. Ocular characteristics associated with the location of focal lamina cribrosa defects in open-angle glaucoma patients. Eye. 2017 Apr;31(4):578-87.

62 Shoji T, Kuroda H, Suzuki M, Baba M, Hangai $\mathrm{M}$, Araie $\mathrm{M}$, et al. Correlation between lamina cribrosa tilt angles, myopia and glaucoma using OCT with a wide bandwidth femtosecond mode-locked laser. PLoS One. 2014;9(12): e116305.

63 Jonas JB, Wang N, Yang D, Ritch R, PandaJonas S. Facts and myths of cerebrospinal fluid pressure for the physiology of the eye. Prog Retin Eye Res. 2015 May;46:67-83. 